\title{
Changing Pattern of Resistance in Typhoid Fever in An Era of Antimicrobial Resistance: Is It Time to Revisit Treatment Strategies?
}

\author{
Sonal Saxena ${ }^{1}$, Ravinder Kaur ${ }^{2}$, Validerjeet Singh Randhawa ${ }^{2}$ \\ ${ }^{1}$ Department of Microbiology, Maulana Azad Medical College, New Delhi, India \\ ${ }^{2}$ Department of Microbiology, Lady Hardinge Medical College, New Delhi, India
}

\begin{abstract}
Objectives: Multi-drug-resistant Salmonella Typhi is a major concern in the current era of antimicrobial resistance. The emergence of ceftriaxone-resistant Salmonella Typhi strains (CRST) has left very few therapeutic options for the treatment of these infections. The objective of this study was to evaluate the typhoid cases diagnosed with CRST and retrospectively analyze the antimicrobial sensitivity pattern of Salmonella Typhi over the past three years.
\end{abstract}

Methods: Laboratory data from our hospital were reviewed for the past three years to examine the trends related to the isolation of Salmonella Typhi from blood and their antimicrobial susceptibility. The case records from the patients whose blood culture detected Ceftriaxone resistant Salmonella Typhi (CRST) were reviewed to assess the clinical condition and outcome.

Results: Analysis of the antimicrobial susceptibility of Salmonella Typhi showed a decline in multidrug-resistant strains. Nalidixic acid resistance has remained high during this period. The resistance to ceftriaxone has shown an increase from $0 \%$ in 2016 to $11(12.6 \%$ ) isolates in 2018. Also, azithromycin resistance has shown a steady increase from $0 \%$ in 2016, $3(3.2 \%)$ in 2017 to 7 (8.5\%) in 2018. There was no clustering of cases by time or space indicating a non-outbreak spread of ceftriaxone resistant strains.

Conclusion: Development of resistance to ceftriaxone and azithromycin necessitates revision of therapeutic choices. All this necessitates an approach to do further extensive surveillance at the community level and improvement in hygiene, sanitation, and drinking water supply. Maybe it is time to revisit the older antibiotics such as ampicillin, cotrimoxazole, and chloramphenicol as therapeutic options for uncomplicated typhoid fever. J Microbiol Infect Dis 2021; 11(1):1-7.

Keywords: Ceftriaxone-resistance S. Typhi, MDR S. Typhi, Typhoid fever, antimicrobial susceptibility

\section{INTRODUCTION}

Enteric fever continues to be a major health problem especially in developing countries such as India. It is estimated that 12 million cases occur annually worldwide [1]. A meta-analysis from India has reported a pooled estimate of incidence at 377 per 1,00,000-person-years with the highest incidence between 2-4 years of age [2].

Enteric fever is further complicated by the development of multidrug resistance strains (strains resistant to ampicillin, cotrimoxazole, and chloramphenicol). The emergence of multidrug resistance strains (MDR) strains saw the treatment options to change to fluoroquinolones. The last few years have seen over-usage of fluoroquinolones for multiple indications. The resultant increase in antimicrobial pressure caused $S$. Typhi strains to become resistant to fluoroquinolones leaving ceftriaxone and macrolides as the last options. Over time, ceftriaxone-resistant Salmonella strains (CRST) have started emerging from various countries including the Indian subcontinent [3]. This is alarming as it leaves very few therapeutic options for the treatment of these infections. Our center caters to a patient population from Delhi and neighboring areas. In 2018 we noticed a number of $S$. Typhi strains resistant to ceftriaxone. This prompted to study of these cases and also retrospectively analyze the antimicrobial sensitivity data of Salmonella Typhi. 


\section{METHODS}

\section{Microbiological methods}

The study was conducted at a 1200 bedded Tertiary care center after due ethical approvals. The microbiology laboratory receives samples from both adults as well as children's hospitals. The blood cultures are performed using automated BacT Alert ${ }^{\mathrm{TM}}$. The isolates were identified and serotyped as per laboratory protocol. The antibiotic sensitivity is performed as per CLSI guidelines using the Kirby Bauer method [4]. All isolates exhibiting multi-drug resistance patterns were subjected to identification and antimicrobial sensitivity analysis by Vitek ${ }^{\mathrm{TM}}$. The laboratory is part of the National AMR surveillance network. As part of the network mandate, any isolate exhibiting an unusual resistance pattern is sent for confirmation to National Referral Center at National Center for Disease Control, Delhi. Quality assurance of laboratory with positive and negative isolates is also carried out at NCDC and at EQAS center of Indian Association of Medical Microbiologists at Sir Gangaram Hospital, New Delhi. The antimicrobial susceptibility data is collated using WHONET ${ }^{\mathrm{TM}}$. The antimicrobial susceptibility data were analyzed using the data analysis tool of WHONET TM for 2016, 2017, and 2018. Blood culture records were reviewed from 2016 to 2018. Laboratory data from our hospital was also reviewed for the past three years to examine the trends related to the isolation of Salmonella Typhi and their antimicrobial susceptibility.

\section{Clinical Data Review}

The case records from the patients whose blood culture detected Ceftriaxone resistant Salmonella Typhi (CRST) were reviewed to assess the clinical condition and outcome. The clinical data were reviewed to assess the outbreak or non-outbreak situation of CRST and to examine and correlate the conditions attributing to such a high level of resistance.

\section{RESULTS}

\section{Microbiological data}

In the study period (between 2016 and 2018) a total of 5,807 bacterial pathogens were isolated from 50,479 blood samples received in the laboratory for culture. An overall culture positivity rate of $11.5 \%$ was observed. Of these 248 isolates of Salmonella Typhi were isolated over a three-year period accounting for $4.3 \%$ of total bacterial isolates (Table1).

Analysis of the antimicrobial susceptibility of Salmonella Typhi (Table 2) isolates showed a decline in multi-drug resistant strains (Strains of S. Typhi resistant to ampicillin, chloramphenicol and trimethoprim sulphamethoxazole) from $9.7 \%$ (7 isolates) in 2016 to $5.8 \%$ (5 isolates) in 2018. Resistance rates to ampicillin and trimethoprim sulphamethoxazole have remained fairly constant over the past three years but the resistance rate to chloramphenicol have decreased from $13.8 \%$ (10 isolates) in 2016 to $9.2 \%$ (8 isolates) in 2018. Amongst fluoroquinolones, nalidixic acid resistance has remained very high in our hospital $(91.8 \%$ in 2016 to $96.5 \%$ in 2018). This makes the treatment options with fluoroquinolones impossible. Overall the past three years have seen a fall in MDR strains along with rising resistance to azithromycin and ceftriaxone in Salmonella Typhi (Figure 1).

A total of 11 isolates showed resistance to ceftriaxone by disc diffusion. Minimum inhibitory concentration of all these isolates was found to be more than 4 by Vitek $^{\mathrm{TM}}$. All these isolates were sensitive to chloramphenicol and meropenem, while all of them were resistant to nalidixic acid. Table 2 shows the antimicrobial susceptibility pattern of ceftriaxone resistant Salmonella Typhi. Comparative analysis of CRST with Salmonella Typhi isolates showed higher resistance to other antibiotics (Figure 2).

\section{Clinical Review of Ceftriaxone Resistant Salmonella Typhi cases}

Analysis of all 11 patient records retrospectively revealed that all cases were unimmunized and had a severe clinical presentation with some complications. They were all admitted for a mean hospitalization period of 28.7 (range 1745) days. All presented with prolonged fever ( $>10$ days) and abdominal pain. Nine patients reported changes in bowel habits, with diarrhea being the most common symptom. Three patients had vomiting and rashes while one patient presented with signs and symptoms of acute abdomen. Seven cases had massive hepatosplenomegaly while all cases had 
pancytopenia (Table 3). All cases had elevated CRP while $8(72 \%)$ had deranged liver enzymes. All these patients lived in different areas of the city. Only two cases were residents of rural areas residing with relatives in Delhi. Also, all cases were reported at different times of a year indicating non-outbreak potential. None of these patients had received typhoid vaccination. One patient died while three cases developed complications. One patient developed septic arthritis. Fluid aspiration from the joint did not reveal any cultivable bacteria. Another patient developed intestinal perforation and was transferred to surgery for management. The third case was complicated by sepsis caused by methicillin-resistant Staphylococcus aureus. The septicemia along with typhoid resulted in the death of this patient.

All cases had prolonged fever which did not respond to multiple antibiotics. Nine out of eleven patients were started on ceftriaxone initially, while two patients were given cefixime. The blood culture report was made available within 72-96 hours still ceftriaxone was continued for a mean of 11.1 days. Six patients with favorable outcomes responded to azithromycin while one case was given meropenem. All patients were prescribed oral cefixime for 10-14 days, while 4 patients were discharged on the same for 10 days.

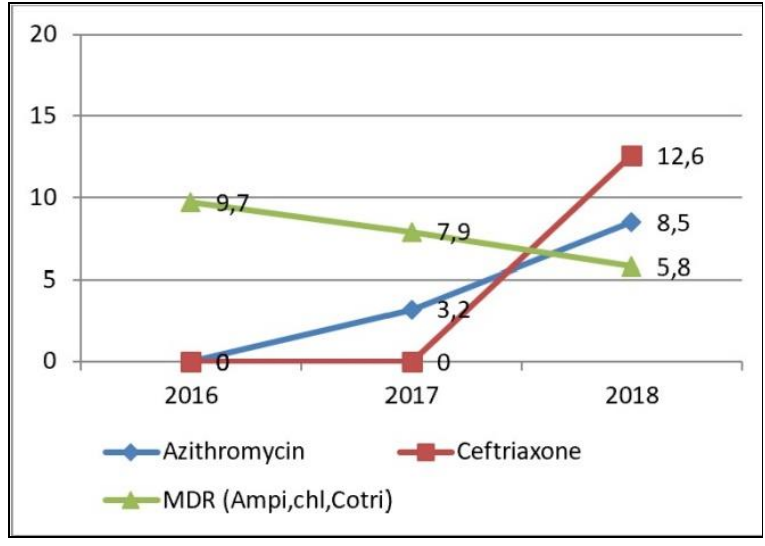

Figure 1. Changing trend of antibiotic sensitivity patterns of Salmonella Typhi (\% resistance).

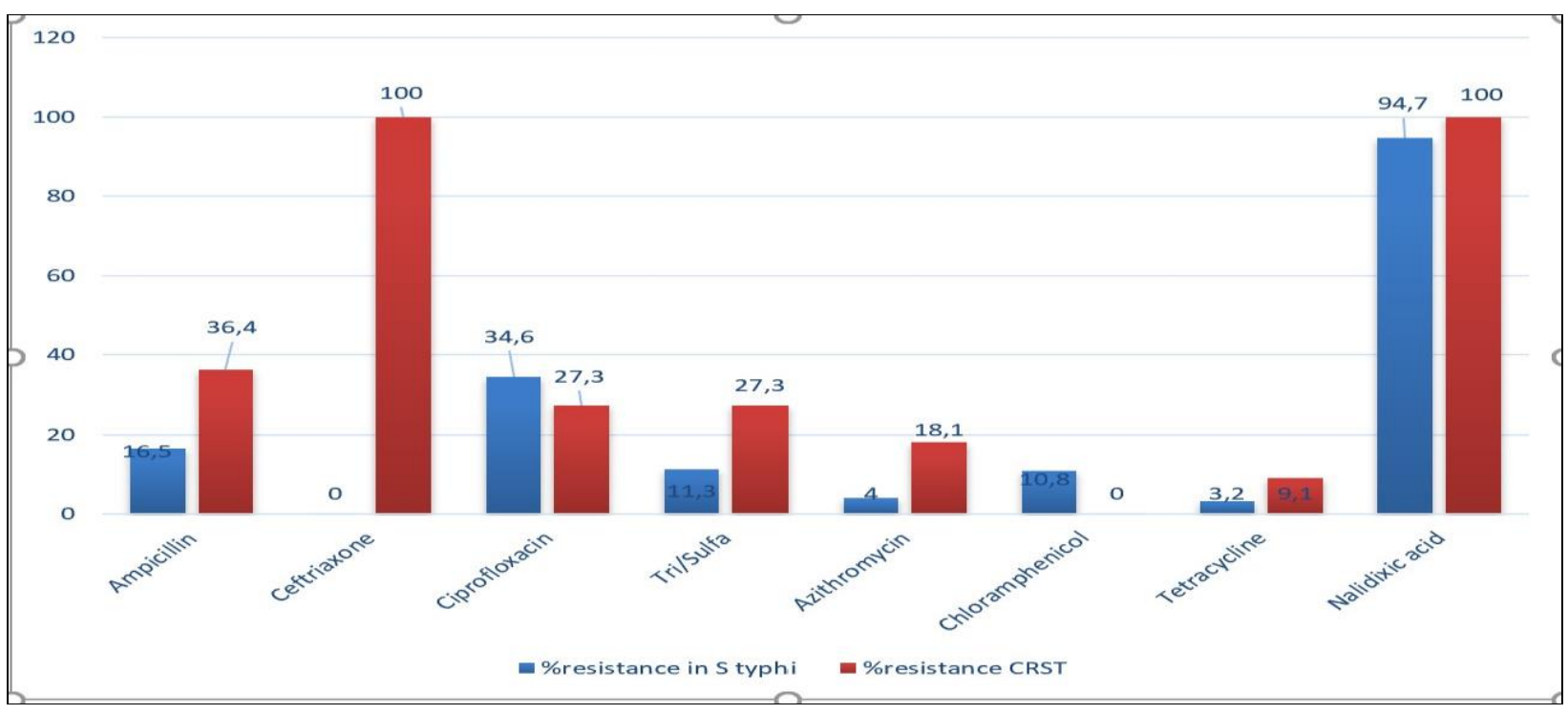

Figure 2. Resistance profile of CRST and Salmonella Typhi.

Table 1. Isolation rates of Salmonella.

\begin{tabular}{lcccc}
\hline Year & $\begin{array}{c}\text { Total number of } \\
\text { blood cultures }\end{array}$ & $\begin{array}{c}\text { Total number of } \\
\text { isolates (\%) }\end{array}$ & S. Typhi (\%) & S. Paratyphi A (\%) \\
\hline 2016 & 15,111 & $1805(12)$ & $72(3.9)$ & $12(0.7)$ \\
2017 & 16,979 & $2178(13)$ & $89(4)$ & $5(0.3)$ \\
2018 & 18,389 & $1824(10)$ & $87(4.7)$ & $18(1)$ \\
Total & 50,479 & $5807(11.5)$ & $248(4.3)$ & $36(0.6)$ \\
\hline
\end{tabular}


Table 2: Resistance pattern of Salmonella Typhi 2016-2108

\begin{tabular}{lccccc}
\hline Antibiotic & $2016, \mathrm{n}=72(\%)$ & $2017, \mathrm{n}=89(\%)$ & $2018, \mathrm{n}=87(\%)$ & $\begin{array}{c}\text { Cumulative } \\
\text { resistance, } \\
\mathrm{n}=248(\%)\end{array}$ & $\begin{array}{c}\text { Resistant } \\
\text { pattern of } \\
\text { CRST }\end{array}$ \\
\hline Ampicillin & $10(13.8)$ & $15(16.9)$ & $16(18.4)$ & $41(16.5)$ & $4(36.4)$ \\
Ceftriaxone & $0(0)$ & $0(0)$ & $11(12.6)$ & $11(4.4)$ & $11(100)$ \\
Ciprofloxacin & $22(30.5)$ & $33(37)$ & $31(35.6)$ & $86(34.6)$ & $3(27.3)$ \\
Trimetoprim/Sulphametaxozol & $7(9.7)$ & $13(14.6)$ & $8(9.1)$ & $28(11.3)$ & $3(27.3)$ \\
Azithromycin & $0(0)$ & $3(3.3)$ & $7(8)$ & $10(4)$ & $2(18.1)$ \\
Chloramphenicol & $10(13.8)$ & $9(10.1)$ & $8(9.2)$ & $27(10.8)$ & $0(0)$ \\
Tetracycline & 0 & 0 & $8(9.2)$ & $8(3.2)$ & $1(9.1$ \\
Nalidixic acid & $66(91.7)$ & $85(95.5)$ & $84(96.5)$ & $235(94.7)$ & $11(100)$ \\
MDR ${ }^{*}$ & $7(9.7)$ & $7(7.9)$ & $5(5.8)$ & $19(7.7)$ & $0(0)$ \\
Cefixime & $0(0)$ & $0(0)$ & $0(0)$ & $3(27.3)$ & $3(27.3)$ \\
\hline
\end{tabular}

*Strains of $S$. Typhi resistant to ampicillin, chloramphenicol and trimethoprim-sulphamethoxazol.

Table 3. Clinical characteristics of Ceftriaxone resistant Salmonella Typhi

\begin{tabular}{|c|c|}
\hline Variables & Number (\%) \\
\hline Age mean (Pediatrics; $n=8$ ) & 7.18 (0.25-14 yrs) \\
\hline Age mean (Adult; $n=3$ ) & 38.66 (28-45 yrs) \\
\hline Duration of fever, mean (range) & $11.3(7-20)$ days \\
\hline Setting urban & $9(81)$ \\
\hline \multicolumn{2}{|l|}{ Complications } \\
\hline Intestinal perforation & $1(9)$ \\
\hline Pancytopenia & $11(100)$ \\
\hline Idiopathic cytopenic purpura & $1(9)$ \\
\hline Septicemia with different bacteria & $3(27)$ \\
\hline Pneumonia & $1(9)$ \\
\hline Massive hepatosplenomegaly & $7(63)$ \\
\hline Septic arthritis & $1(9)$ \\
\hline \multicolumn{2}{|c|}{ Antibiotics prescribed (Mean days of therapy \& range) } \\
\hline Ceftriaxone & $9(11.1\{10-17$ days $\})$ \\
\hline Meropenem & 4 (8.5 $\{5-14$ days $\})$ \\
\hline Azithromycin & $6(11.6\{8-14$ days $\})$ \\
\hline Cefixime & $11(12.4\{10-14$ days $\})$ \\
\hline Doxycycline & 1 (7 days) \\
\hline
\end{tabular}

\section{DISCUSSION}

Over the past few years, there has been a decline in the isolation rates of MDR Salmonella Typhi [3,5-8]. At our center, the isolation rates of multidrug-resistant Salmonella Typhi have shown a decline from $9.7 \%$ in 2016 to $5.8 \%$ in 2018. Trend analysis from Vellore has shown a decline from $50 \%$ to $5 \%$ over a period of 15 years from 2000 to 2015 [3]. MDR strains 
accounted for $5 \%$ of all S. Typhi analyzed in 2017 by the ICMR surveillance network (6). Earlier Chande et al. from Central India in 2002 have also reported a low isolation rate in MDR isolates (22\%) [7]. A study from Delhi also found a reduction in MDR rates from $36 \%$ in 1999 to $8 \%$ in 2008 [8].

Another systemic review from Asia has reported a decline in MDR strains in Asia to $<20 \%$ in 2011-2015 while resistance to nalidixic acid and fluoroquinolone continued to increase from $20 \%$ in $2001-2005$ to $65 \%$ in $2011-2015$ [5]. A study from Indonesia has also reported MDR rates below $5 \%$ [9]. A retrospective study from Nepal in 2017 has reported a decline in MDR strains over a period of 23 years [10). Studies from Indonesia, Bangladesh, and Vietnam in 2017 have reported low MDR rates of $1.8 \%$ to $4.3 \%$ over a span of five years [9,11-13].

Ampicillin resistance rates have remained below $20 \%$ at our center. Studies from India have reported highly variable rates of ampicillin resistance ranging from $3.7 \%$ to $34.1 \%[3,14$ 18]. A study from Delhi in 2006 has reported a $98.2 \%$ sensitivity rate to ampicillin [19]. Another study from Delhi has reported a decline in ampicillin resistance rates from $36 \%$ in 1999 to $8 \%$ in 2008 [8].

Resistance rates against trimethoprimsulphamethoxazole and chloramphenicol have remained around $10 \%$ in the past three years. Most studies from India have reported similar rates [15,21]. Resistance rates of $3.6 \%$ against chloramphenicol and $30.4 \%$ against trimethoprim-sulphamethoxazole have been reported from Delhi in 2006 [19]. A reduction in chloramphenicol resistance rates from $10 \%$ in 2008 to $36 \%$ in 1999 has been reported by Raveendran et al. from Delhi. They also reported a decrease in resistance to trimethoprim-sulphamethoxazole from $36 \%$ to $24 \%$ ]8]. Patel et al. from Varanasi have reported resistance of $19.1 \%$ to trimethoprimsulphamethoxazole and $2.1 \%$ to chloramphenicol in 2011-13 [21]. ICMR surveillance network data for 2017 reported resistance rates of $4 \%, 7.6 \%$, and $22.5 \%$ to chloramphenicol, ampicillin, and trimethoprimsulphamethoxazole respectively [6].

In our study, we found $>90 \%$ of isolates were resistant to nalidixic acid-resistant Salmonella
Typhi (NARST). Resistance to ciprofloxacin has also remained above $30 \%$. Similar trends have been reported by other authors as well. Variable but high resistance rates to nalidixic acid have been reported from India ranging from $51 \%$ to $100 \%[8,15-18,22]$. ICMR surveillance network reported ciprofloxacin and pefloxacin resistance at $37 \%$ and $73.3 \%$ respectively in 2017 [6].

In the past few years, resistance to 3rd generation cephalosporin has increased in Asia from $1.5 \%$ in $2006-2010$ to $4 \%$ in 2011-2015 [5]. Sporadic cases of azithromycin and ceftriaxoneresistant S. Typhi have been reported from Pakistan, Bangladesh, and India $[3,21,25,26]$. Ceftriaxone-resistant Salmonella Typhi was initially detected in Hyderabad, Pakistan [27]. ICMR surveillance network reported $0.4 \%$ isolates resistant to ceftriaxone [6). Raza et al. from Nepal reported $4.3 \%$ in 2012 while another study from Vellore in 2015 reported $2.4 \%$ isolates resistant to ceftriaxone $[3,20]$. Chandane et al. have reported the CRST rate of $9.3 \%$ in Mumbai while another study from Gangetic plains reported $23.4 \%$ resistance to ceftriaxone [21].

S. Typhi isolates resistant to azithromycin have been reported from South Africa and Bangladesh in the past two years $[26,29]$. Our study has also found a rising trend in azithromycin resistance (Figure 2). Patel et al. in 2017 have reported $21.3 \%$ isolates resistant to azithromycin from Varanasi [21].

Cases of typhoid fever caused by CRST have been associated with more complications, prolonged antibiotic treatments, and more duration of hospitalizations thereby increasing the costs [25]. Similar trends were observed in or study also.

Patient demographics like age, sex, and clinical presentation of our cases were found to be similar to Qamar et al. and Yousafzai et al. $[25,27]$. The mean duration of fever was found to be for 14 days by Yousafzai et al. in contrast to 11.3 days in our study [25]. Lack of any relevant travel history, absence of any clustering over time and space indicated that these cases were not part of an outbreak but seem to be sporadically present in the community. This is in sharp contrast to earlier studies that have reported CRST as an outbreak $[25,27]$. 


\section{Conclusion}

The emergence of strains resistant to ceftriaxone and azithromycin clearly indicates the time to revisit our treatment strategies. Infection with such strains predisposes patients to develop complications and will need treatment with newer antibiotics.

\section{ACKNOWLEDGMENTS}

Declaration of Conflicting Interests: The authors declare that they have no conflict of interest.

\section{Funding: Not applicable \\ REFERENCES}

1. Buckle GC, Walker CL, Black RE Typhoid fever and paratyphoid fever: Systematic review to estimate global morbidity and mortality for 2010 . J Glob Health 2012 2: 010401.

2. John J, Van Aart CJC, Grassly NC. The Burden of Typhoid and Paratyphoid in India: Systematic Review and Meta-analysis. PLoS Negl Trop Dis 2016;10(4): e0004616.

3. Balaji V, Kapil A, Shastri J, et al. Longitudinal Typhoid Fever Trends in India from 2000 to 2015. Am J Trop Med Hyg 2018; 99(S3):34-40.

4. Performance standards for antimicrobial sensitivity testing. Clinical and Laboratory Standards Institute M10025S.2015

5. Britto CD, Wong VK, Dougan G, Pollard AJ A systematic review of antimicrobial resistance in Salmonella enterica serovar Typhi, the etiological agent of typhoid. PLoS Negl Trop Dis 2018;12(10): e0006779.

6. AMR surveillance network Indian Council of Medical Research 2017. Annual report Antimicrobial Resistance Surveillance Network January 2017-December 2017. Available at https://www.icmr.nic.in/sites/default/files/reports/an nual_report_amr_jan2017-18.pdf. ICMR 2017

7. Chande C, Shrikhande S, Kapale S, Agarwal S, Fule RP. Change in antimicrobial resistance pattern of Salmonella Typhi in central India. Indian J Med Res 2002; 115:46-8.

8. Raveendran R, Datta S, Wattal C. Drug resistance in Salmonella Enterica serotype Typhi and Paratyphi A. JIMSA 2010;23(1): 21-24.

9. Lugito NPH, Cucunawangsih. Antimicrobial Resistance of Salmonella enterica Serovar S. Typhi and Paratyphi Isolates from a General Hospital in Karawaci, Tangerang, Indonesia: A Five-Year Review. Int J Microbiol 2017, 2017:6215136.

10. Zellweger RM, Basnyat B, Shrestha $P$, et al. (2017) A 23-year retrospective investigation of
Salmonella Typhi and Salmonella Paratyphi isolated in a tertiary Kathmandu hospital. PLoS Negl Trop Dis 11(11): e0006051.

11. Punjabi $\mathrm{NH}$, Agtini MD, Ochiai RL, et al. Enteric fever burden in North Jakarta, Indonesia: a prospective, community-based study. J Infect Dev Ctries 2013; 7:781-787.

12. Chiou CS, Lauderdale TL, Phung DC, et al. Antimicrobial resistance in Salmonella enterica serovar Typhi isolates from Bangladesh, Indonesia, Taiwan, and Vietnam Antimicrob Agents Chemother 2014;58(11):6501-7.

13. Alam A Pola resistensi Salmonella enterica Serotipe typhi, departemen IImu Kesehatan anak RSHS, Tahun 2006-2010 Sari Pediatri 2011;12(5):296-301.

14. Yaxian J, Hui $Z$, Hua $N$, et al. Antimicrobial resistance surveillance of Salmonella isolates from the First People's Hospital of Yunnan Province, China J Infect Develop Ctries 2015;9(4):333-337

15. Qamar FN, Azmatullah A, Kazi AM, Khan E, Zaidi AKM A three-year review of antimicrobial resistance of Salmonella enterica serovar S. Typhi and Paratyphi A in Pakistan J Infect Develop Ctries 2014; 8(8): 981-986.

16. Bhattacharya SS, Das U, Choudhury BK. Occurrence \& antibiogram of Salmonella Typhi \& S. Paratyphi A isolated from Rourkela, Orissa. Indian J Med Res 2011;133: 431-433.

17. Menezes GA, Harish BN, Khan MA, Goessens $\mathrm{WH}$, Hays JP Antimicrobial resistance trends in blood culture positive Salmonella Typhi isolates from Pondicherry, India, 2005-2009. Clin Microbiol Infect 2012; 18: 239-245.

18. Dutta S, Das S, Mitra U, et al. Antimicrobial resistance, virulence profiles and molecular subtypes of Salmonella enterica serovar S. Typhi and Paratyphi A blood isolates from Kolkata, India during 2009-2013. PLoS One 2014; 9: e101347.

19. Muthu G, Suresh A, Gnadesikan S, Ramesh S Studies on antimicrobial susceptibility pattern of Salmonella isolates from Chennai, India. Int $\mathrm{J}$ Pharma Bio Sci2011; 2: 435-442.

20. Jain S, Das Chugh T, 2013. Antimicrobial resistance among blood culture isolates of Salmonella enterica in New Delhi. J Infect Dev Ctries 2013:7: 788-795.

21. Manchanda V, Bhalla $P$, Sethi $M$, Sharma V K. Treatment of enteric fever in children on the basis of current trends of antimicrobial susceptibility of Salmonella enterica serovar typhi and paratyphi A. Indian J Med Microbiol 2006;24:101-106

22. Raza S, Tamrakar R, Bhatt CP, Joshi SK. Antimicrobial susceptibility patterns of Salmonella Typhi and Salmonella paratyphi A in a tertiary care 
hospital. J Nepal Health Res Counc 2012; 10: 214-217.

23. Patel SR, Bharti S, Pratap CB, Nath G. Drug Resistance Pattern in the Recent Isolates of Salmonella Typhi with Special Reference to Cephalosporins and Azithromycin in the Gangetic Plain. Journal of Clinical and Diagnostic Research. 2017;11(6): DM01-DM03.

24. Chand HJ, Rijal KR, Neupane B, Sharma VK, Jha $B$. Re-emergence of susceptibility to conventional first line drugs in Salmonella isolates from enteric fever patients in Nepal J Infect Develop Ctries 2014;8(11): 1483-1487.

25. Singhal L, Gupta PK, Kale P, Gautam V, Ray P Trends in antimicrobial susceptibility of Salmonella Typhi from north India (2001-2012). Indian J Med Microbiol 2014 32: 149-152.

26. Kundu R, Ganguly N, GhoshTK, Yewale VN, Shah RC, Shah NK. IAP Task Force Report: Management of Enteric Fever in Children. Indian Pediatrics 2006; 43:884-887.

27. Upadhyay R, Nadkar MY, Muruganathan A, et al. API Recommendations for the Management of Typhoid Fever. J Assoc Phys India; 63:77-96.

28. Yousafzai MT, Qamar FN, Shakoor $S$ et al. Ceftriaxone-resistant Salmonella Typhi Outbreak in Hyderabad City of Sindh, Pakistan: High Time for the Introduction of Typhoid Conjugate Vaccine. Clin Infect Dis 2019; 68: S16-S21.

29. Ahsan S, Rahman S. Azithromycin Resistance in Clinical Isolates of Salmonella enterica Serovar S. Typhi and Paratyphi in Bangladesh Microbial Drug Resis 2019;25(1):8-13.

30. Qamar FN, Yousafzai MT, Khalid M Outbreak investigation of ceftriaxone-resistant Salmonella enterica serotype Typhi and its risk factors among the general population in Hyderabad, Pakistan: a matched case-control study. Lancet Infect Dis 2018; 18: 1368-76.

31. Chandane P, Gandhi A, Bowalekar S. Study of antibiotic susceptibility pattern of Salmonella Typhi in children suffering from enteric fever. Ann Trop Med Public Health 2017; 10:440-443.

32. Keddy KH Smith AM Sooka A et al. The burden of typhoid fever in South Africa: the potential impact of selected interventions. Am J Trop Med Hyg 2018; 99: 55-63. 